\title{
ANÁLISIS DEL PROCESO DE PRODUCCIÓN DE UNA LÍNEA DE COMPONENTES DE TURBINAS DE AVIÓN
}

\author{
RAFAEL GRANADOS GIL* \\ https://orcid.org/0000-0003-4874-9187 \\ ILEANA MONSREAL BarRera* \\ https://orcid.org/0000-0003-4567-9830 \\ JUAN JOSÉ BARRERA GUTIÉRREZ* \\ https://orcid.org/0000-0002-3098-4667 \\ Universidad Autónoma de Yucatán, México
}

Recibido: 18 de febrero del 2021 / Aprobado: 15 de julio del 2021

doi: https://doi.org/10.26439/ing.ind2021.n41.5540

RESUMEN: La particular situación de la industria aeronáutica para los próximos años obligará a las plantas productoras vinculadas con ésta a analizar sus procesos productivos buscando, entre otras cosas que podrían destacarse, mayor flexibilidad y eficiencia. El presente artículo analiza el proceso de manufactura de una línea de producción de componentes de turbinas de avión que enfrentaba problemáticas en su flujo y capacidad de producción, por lo que se plantean propuestas realistas y acordes al caso que permitan mejoras en su capacidad en un futuro cercano. En este estudio se utilizó la metodología aplicada por Okutmuş et al. (2015) para la recolección de datos y, posteriormente, para el análisis de la información, se recurrió a la metodología utilizada por Molano y Materón (2018). Finalmente se concluye que se podría incrementar la capacidad de producción. Por ello, se presentan propuestas realistas sobre el caso, que permiten mejorar su capacidad en un futuro próximo.

PALABRAS CLAVE: capacidad de producción / procesos / tiempo de ciclo / industria aeronáutica

\footnotetext{
*Correos electrónicos: rafaelgranados93@outlook.com; ileana.monsreal@correo.uady.mx; juanjbgz2@outlook.com
} 


\section{PROCESS ANALYSIS OF A PRODUCTION LINE OF AIRCRAFT TURBINE COMPONENTS}

ABSTRACT. The particular situation of the aeronautical industry in the coming years will force related manufacturing plants to analyze their production processes seeking, among other things that could be remarkable, greater flexibility and efficiency. This article analyzes the manufacturing process of a production line of aircraft turbine components, which faced flow and production capacity problems. The study used the methodology applied by Okutmuş et al. (2015) for data collection, and subsequently, resorted to the methodology used by Molano \& Materón (2018) to analyze of the information. Finally, it concludes that production capacity could be increased. Therefore, realistic proposals on the case are presented, which allow improvements in its capacity in the near future.

KEYWORDS: production capacity / processes / cycle time / aeronautical industry 


\section{INTRODUCCIÓN}

En 2018 la industria aeronáutica se encontraba en una etapa importante de crecimiento y desarrollo ya que se esperaba que entre el 2017 y el 2036 hubiera un incremento en el sector del transporte aéreo de un 83,6 \% (AIRBUS, 2018), lo que se traducía en una demanda de 35000 aviones en los próximos veinte años. Sin embargo, dada la situación actual debida a los efectos de la pandemia que se está viviendo desde el 2020 y de acuerdo con Semprún (2021), las aerolíneas de todo el mundo han reducido un 20 \% su flota en servicio y frenado la compra y recepción de nuevos aviones por el desplome de la demanda, que tardará tres años más en superar los efectos del COVID-19, retrasando así a 2026 la recuperación total de la industria aeronáutica.

Esta situación supone un gran reto para las plantas productoras de esta industria, que se verá obligada a reducir sus niveles de producción, pero con la posibilidad latente de reactivación posterior, por lo que requerirá procesos productivos más eficientes, flexibles y de menor costo que los que actualmente tiene.

Es así como, considerando la paradójica situación planteada, se hace necesario abordar la mejora de los procesos productivos de una industria que da trabajo a una gran cantidad de personas en el mundo; tan solo para citar un ejemplo, en Baja California, México, según De León (2021) citando al Gobierno del Estado, al presente año se estima que existen cien empresas, que dan trabajo a alrededor de 33000 personas, cuya supervivencia está ligada a la microeconomía de este sector.

El presente artículo es un acercamiento al sector aeronáutico mediante el análisis del proceso de un estudio de caso; sin que pretendamos ser exhaustivos, consideramos que los estudios de caso, particularmente ligados a actividades productivas, son claves para entender situaciones que pueden servir para evitar en otras organizaciones los mismos errores o bien comprender mejor las causas y sus consecuencias, tal como sucede en la medicina, en la que muchas veces se parte del estudio de un paciente que presenta alguna enfermedad o trastorno para posteriormente acumular diversos casos que lleven a conclusiones que en un inicio no se vislumbraban.

Son varios los trabajos que han abordado el análisis de procesos para detallar sus efectos en las organizaciones; los efectos que los procesos puedan traer a la industria van desde ahorro en insumos hasta la mejora de procesos; entre las referencias que se consideraron para el desarrollo de la metodología con la que se trabajó, se encuentran:

Ortiz-Triana y Caicedo-Rolón (2015) concluyeron que los problemas relacionados con la capacidad se presentan por la existencia de cuellos de botella causados por equipos y falta de personal operativo, así como también por una mala programación de actividades. 
Serrano-Gómez y Ortiz-Pimiento (2012) realizaron una revisión de la literatura sobre los diferentes modelos para el mejoramiento de procesos con la finalidad de alinear las operaciones de las empresas con sus prioridades estratégicas.

Luna-Puente et al. (2010) abordaron el tema en la industria textil al realizar un análisis del proceso de fabricación de cuellos para playeras tipo polo, con la finalidad de disminuir las pérdidas ocasionadas por el desperdicio de hilo.

Arango-Serna et al. (2015) aplicaron la metodología Kanban y el análisis de su efecto en una empresa de fabricación de transformadores. Mediante la aplicación de su propuesta fueron capaces de mejorar la programación de la producción y redujeron la cantidad de producto en proceso no utilizado, de forma que fue posible reducir el inventario.

De igual forma, Robles (2012) realizó una propuesta de mejora en el proceso productivo de una empresa dedicada a la producción de cereales, mediante la implementación del Lean Manufacturing; con ello se logró identificar las deficiencias del proceso, disminuir los tiempos de producción y generar menos merma y ahorros económicos para la empresa.

Autores como Rocha, Pinto y Silva (2018) trabajaron con la filosofía Lean en una empresa de manufactura de joyería y aplicaron diferentes herramientas como los sistemas Pull, Kanban, 5 S's, entre otros, para impulsar la efectividad y la eficiencia de la operación de la planta y lograr disminuir productos defectuosos, inventarios excesivos, problemas de transporte y tiempos de espera.

Molano y Materón (2018) realizaron un análisis de los procesos en una empresa de alimento para animales con la finalidad de identificar los principales factores que afectan la productividad y plantearon propuestas para aumentarla, así como para reducir los tiempos de ciclo.

La teoría de restricciones cobra importancia al ser una metodología que se ha utilizado ampliamente en todo tipo de industrias, tanto públicas como privadas, como lo señalan Okutmuș et al. (2015), quienes aplicaron esta teoría en la industria Mueblera para identificar las restricciones en el proceso productivo mediante la metodología que Goldratt propuso en 1984.

Tao et al. (2018) describen la creciente cantidad de información generada por las empresas hoy en día y enfatizan la importancia del análisis de la misma, mediante herramientas de análisis estadístico y Big Data, que permitan incrementar la eficiencia de la industria manufacturera, así como aportar sustento a la toma de decisiones, supervisión de operaciones y equipo, control de calidad, predicción de fallas y mantenimiento inteligente.

Sin embargo, es poca la información sobre procesos productivos de la industria aeronáutica y es, en ese sentido, que cobran importancia trabajos como el presente que, 
abordado como estudio de caso, se acerca al planteamiento de Hernández-Pólito (2013) que plantea una estrategia de investigación cuya información resultante puede coadyuvar en la toma de decisiones sobre situaciones "fuera de lo generalmente esperado" en la conducción de las organizaciones. Es una estrategia guiada por tres preguntas de investigación centrales: ¿Cuándo? ¿Cómo? y ¿Por qué? La premisa es la necesidad de entender en profundidad el caso. La investigación mediante el método de los estudios de caso permite la exploración y comprensión de las situaciones complejas que se enfrentan y, esencialmente, cuando encontramos que las ideas y conceptos de la teoría de la administración no operan bajo ciertas condiciones.

Como ya se mencionó, el presente artículo aborda uno de esos complejos procesos de la industria aeronáutica; el principal objetivo del trabajo fue analizarlo y plantear propuestas realistas y acordes al caso de una línea de productos (que denominaremos NG por razones de confidencialidad) que permitan en un futuro cercano aumentar la capacidad de producción de dichos componentes, mediante la disminución del tiempo de ciclo de proceso. Una vez detectadas las causas, se presentan las propuestas de mayor impacto que ayudarían a reducir o eliminar las trabas encontradas. Cabe comentar que, aunque el estudio implicó gran profundidad en el análisis, la alta dirección de la empresa consideró que hay información que no debe ser pública pues puede afectar a la empresa o afectar los intereses de sus clientes, razón por la cual se tuvo particular cuidado en mantener el nombre, los datos, las cifras y los cálculos que ellos consideraron delicados, fuera de la presente publicación.

La lista de problemas que afectaban la capacidad de producción en la planta objeto de estudio se resume a continuación:

- Mala programación específica de la producción: no se conocían las capacidades exactas de los equipos y procesos.

- Recurso humano ocioso.

- Problemas de flujo de materiales.

- Equipos detenidos por calibraciones vencidas.

Así también cabe comentar que, al iniciar el trabajo, la planta no contaba con un estándar de medición de la productividad de todo el sistema. El único parámetro de medición consistía en cubrir diariamente el equivalente a un número determinado de horas estándar de trabajo (ESH: earned standard hours en inglés). Este número no era fijo y aumentaba cada mes, según lo determinaba la gerencia y el estamento corporativo de la empresa. A cada producto o número de parte se le asignaba un valor en ESH, determinado por la industria estadounidense, que oscilaba entre 0,1 y 5 horas por pieza, dependiendo del grado de complejidad del producto, ya que, a este valor se le atribuía el tiempo efectivo invertido 
a cada pieza. La planta iba sumando tiempo estándar al finalizar el procesamiento de cada pieza a través de todas las operaciones de su ruta de trabajo.

Adicionalmente, al iniciar el proyecto, observamos en la planta una cierta conformidad con la producción y el modo de operar, dado que, siendo una maquiladora, mucho se definía en las oficinas centrales y no se detectaba preocupación por su proceso productivo a pesar de situaciones que evidenciaban fallas importantes.

\section{METODOLOGÍA}

El enfoque de este trabajo fue mixto, ya que incorpora elementos de enfoques tanto cualitativos como cuantitativos.

Se presenta un tipo de diseño preexperimental, específicamente de un caso de estudio único. Este tipo de diseño permite tomar la realidad de un evento ocurrido en una organización y que es idóneo para investigar, ya que sirve para explicar relaciones causales complejas, estudiar cambios a través del tiempo, elaborar perfiles descriptivos y generar teorías o generalizaciones teóricas utilizando una perspectiva holística del fenómeno estudiado, entendiendo el contexto real en el que se desarrollaron los hechos (Saavedra García, 2017).

Cómo investigadores se aplicó la técnica de observación-participación, en la que el investigador baja al campo, se adentra en el contexto social que quiere estudiar, vive cómo y con las personas objeto del estudio, comparte con ellas la cotidianidad, les hace preguntas, descubre sus preocupaciones y sus esperanzas, sus concepciones del mundo y sus motivaciones al actuar, con el fin de desarrollar esa visión desde dentro tan importante para la comprensión (Corbetta, 2007).

Usando como referencia la metodología que aplicaron Okutmuş et al. (2015) para el análisis del proceso y Molano y Materón (2018) para la reducción de tiempos de ciclo, se utilizó la metodología presentada a continuación.

Etapa 1: Diagnóstico de la situación mediante el análisis de los tiempos de ciclo de la línea de productos NG.

1.1 Descripción del proceso.

1.2 Identificación de los tiempos de ciclo.

1.3 Determinación de la capacidad máxima de producción.

1.4 Determinación del tiempo de ciclo máximo permisible.

Etapa 2: Identificación de las principales causas de ineficiencia en la operación.

2.1 Identificación preliminar de las principales causas. 
2.2 Definición del grado de afectación de cada una de las causas identificadas.

Etapa 3: Propuestas de solución que permitan mejorar el flujo de productos e incrementar la capacidad de producción en un futuro cercano.

\section{RESULTADOS}

\section{ETAPA 1. Descripción del proceso de producción}

La secuencia general del proceso de manufactura de los productos NG se muestra en la figura 1.

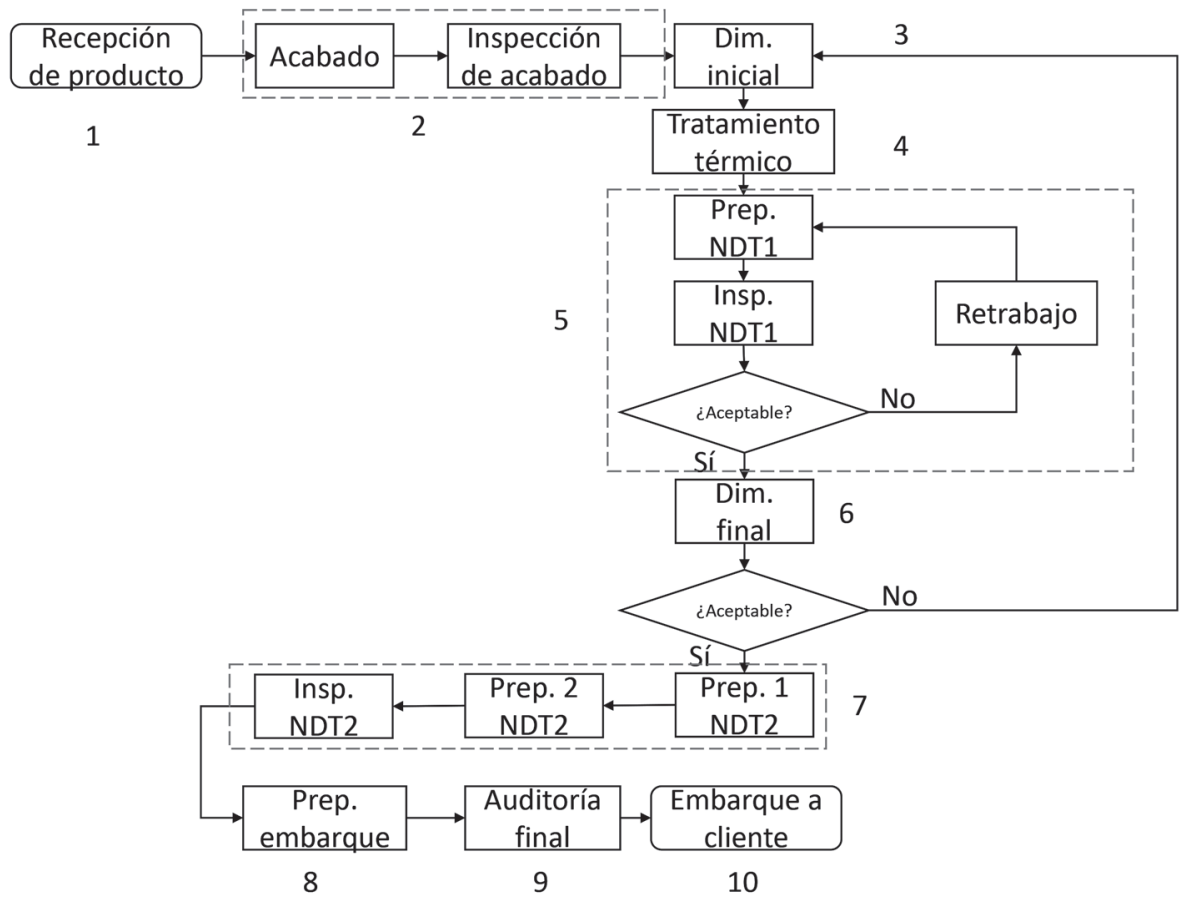

Figura 1. Proceso

Elaboración propia

Como se observa en la secuencia presentada en la figura 1, en todo el proceso de manufactura intervienen una larga serie de operaciones e inspecciones. Aquellas operaciones marcadas con líneas punteadas son las que conllevan un mayor tiempo. A continuación, se describe brevemente el proceso de manera general:

- Recepción de producto: en esta etapa inicia el proceso en la planta. Todos los días, las plantas de fundición de Estados Unidos envían producto a México por 
avión para su procesamiento. El producto llega en cajas a la planta y es descargado de los vehículos del transportista para ser ubicado en el área de entrada de producto denominada incoming o recepción; aquí el producto espera hasta ser programado en las operaciones de acabado para iniciar su procesamiento. Dicha área cuenta con un auxiliar de producción encargado de ordenar y clasificar el producto tan pronto llega para facilitar su ubicación por cliente y número de parte. Una vez realizada la programación del trabajo, es el auxiliar el encargado de llevar el producto al área de trabajo para que el personal operativo pueda trabajar las piezas asignadas durante el turno.

- Trabajo de acabado e inspección: Esta es una de las operaciones principales del proceso, consiste en eliminar los principales defectos presentes en la superficie de la pieza, procedentes del proceso de fundición. Estos defectos son removidos mediante el uso de materiales abrasivos. Permiten eliminar rebabas, irregularidades superficiales como porosidad, entre otros defectos del proceso de fundición. Existen defectos no removibles, característicos del proceso de fundición que comprometen la integridad de la pieza en funcionamiento, por lo que estas piezas son rechazadas desde un inicio. La finalidad de esta operación es obtener una pieza visualmente aceptable según las especificaciones de cada cliente. Una vez que la pieza obtiene la conformidad, es aceptada y se mueve a la siguiente operación.

- Inspecciones dimensionales: Se realiza una inspección dimensional inicial (preliminar) y una inspección final. Esta operación consiste en la medición de ciertas características de las piezas mediante la utilización de diferentes herramientas y equipos de medición, para corroborar que sus dimensiones corresponden a las que cada cliente especifica en sus planos. Al detectarse algún defecto en la pieza, esta se debe reparar para lograr que la medición se inscriba en el rango de tolerancia. Las reparaciones pueden ir desde desgastar un punto de la pieza mediante el uso de abrasivos como lijas o chorros de arena hasta torcer o doblar alguna característica de la pieza. Es importante recalcar que cada número de parte cuenta con un equipo específico que solo puede usarse para ese determinado producto. La inspección final es la verificación de las piezas medidas y reparadas en la medición inicial. Consiste en la medición completa de todas las características que el cliente requiere para la aceptación de la pieza. Si la pieza es conforme, se acepta y se mueve a la siguiente operación, si es rechazada, la pieza tiene que ser retrabajada. Las operaciones inicial y final se realizan en la misma área y utilizan los mismos equipos, por lo que estas operaciones comparten recursos entre sí.

- Tratamiento térmico: Esta operación consiste en ciclos controlados de calentamiento y enfriamiento de las piezas metálicas con la finalidad de cambiar 
sus propiedades físicas y mecánicas. Usualmente se utiliza para aumentar la fuerza y resistencia del material, aunque también pude ser utilizada para alivio del estrés mecánico interno del material, para tener como resultado una pieza dimensionalmente estable, menos propensa a tener variaciones en sus dimensiones debido a la redistribución del estrés residual (Engineers Edge, 2018).

- Pruebas no destructivas (NDT): Las pruebas no destructivas consisten en diferentes evaluaciones e inspecciones que se realizan a las piezas para identificar defectos que no son detectables a simple vista, pero sin afectar la integridad del material evaluado. Las pruebas no destructivas realizadas en la planta se llevan a cabo con la finalidad de detectar defectos no apreciables a simple vista como grietas, daños internos, y otras fallas características del proceso de fundición.

- Preparación para embarque: Esta es la última etapa de los procesos realizados en la planta y consiste en la aplicación de un número de serie único a cada pieza (para dar trazabilidad al producto una vez que las piezas han salido de la planta) y su posterior empaque final.

- Auditoría final: Es una auditoría previa al embarque del producto al cliente mediante el muestreo de determinado número de piezas, dependiendo de la cantidad que se requiera enviar al cliente (al menos un $20 \%$ ) y puede realizarse de manera diaria o semanal. Se inspeccionan diferentes características de la pieza de manera aleatoria. Si la auditoría se acepta, se libera el producto para embarque; si se rechaza, se inspecciona todo el lote al $100 \%$ hasta confirmar que el problema se ha corregido.

- Embarque al cliente: Una vez que el producto está terminado y ha sido auditado, este queda liberado y disponible para ser enviado directamente al cliente. El producto empacado es llevado al almacén de productos terminado en donde, de acuerdo con los requerimientos del cliente, es embalado y puesto en tarimas para su recolección y envío al cliente.

\section{Descripción del problema}

La figura 2 muestra la demanda proyectada por el cliente para la línea de productos NG para los próximos años. Como se puede observar, entre el 2018 y el 2025 se planeaba incrementar la demanda en más de un $85 \%$. 


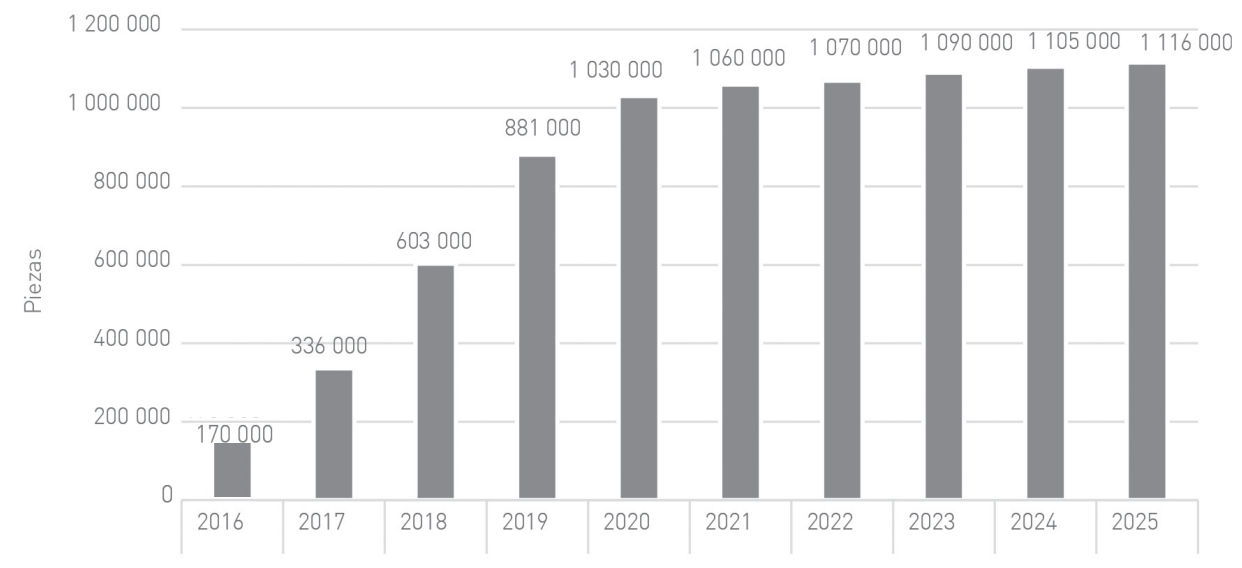

Figura 2. Gráfica de la proyección de demanda de los productos NG Elaboración propia a partir de la información del cliente

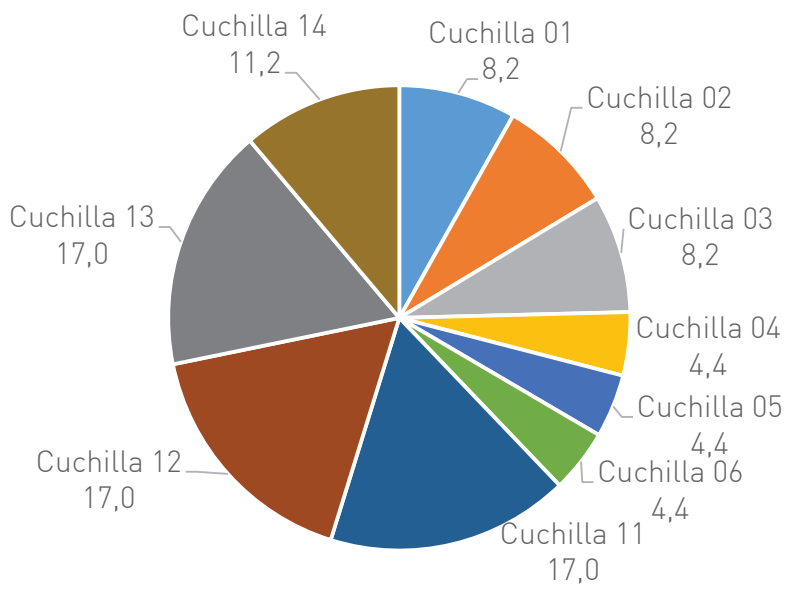

Figura 3. Gráfica del desglose de la demanda por producto de la familia NG (porcentajes) Elaboración propia

La familia de productos NG está conformada por 10 productos diferentes utilizados en dos motores distintos. La proporción de la demanda por número de parte se muestra en la figura 3.

La planta no contaba con un estándar de medición de productividad para todo el sistema. El único parámetro que se tenía era el de cubrir diariamente el equivalente a un número determinado de horas estándar de trabajo (ESH: earned standard hours), este 
número no era fijo y cada mes aumentaba, según decisiones de la gerencia y del estamento corporativo de la empresa. Cada producto contaba con un valor ESH asignado por las plantas de Estados Unidos, el cual oscilaba entre 0,1 y 5 horas por pieza. Las piezas de mayor volumen contaban con valores asignados como se observa en la tabla 1.

Tabla 1

Valor en ESH por producto NG (asignados por las plantas de EE. UU)

\begin{tabular}{cc}
\hline Producto & Horas estándar \\
\hline Cuchilla 01 & 0,45 \\
Cuchilla 02 & 0,45 \\
Cuchilla 03 & 0,50 \\
Cuchilla 04 & 0,61 \\
Cuchilla 05 & 0,52 \\
Cuchilla 06 & 0,57 \\
Cuchilla 11 & 0,456 \\
Cuchilla 12 & 0,502 \\
Cuchilla 13 & 0,50 \\
Cuchilla 14 & 0,73 \\
\hline
\end{tabular}

Elaboración propia

Fuera de esto, no se contaba con otro indicador que reflejara alguna medida de productividad de la planta ya que se desconocían los tiempos reales invertidos en el procesamiento de cada producto. Para conocer los tiempos reales de producción de cada producto, se consultó la base de datos de la empresa (por razones de confidencialidad no se proporciona el detalle) con ayuda del programa SQL Server Management Studio. En él se consultó el tiempo medio que transcurría entre el momento en que una pieza era liberada de una operación y era procesada en la siguiente, es decir, el tiempo que espera cada pieza desde que es puesta en cola para ser procesada en la siguiente operación hasta que finaliza su procesamiento en dicha operación; se consideraron todas las operaciones correspondientes al proceso respectivo. Con los datos obtenidos se construyó la tabla 2. 
Tabla 2

Tiempo real promedio para completar el procesamiento de una pieza

\begin{tabular}{cc}
\hline Producto & $\begin{array}{c}\text { Tiempo real de producción por producto } \\
\text { (horas) }\end{array}$ \\
\hline Cuchilla 01 & 124,77 \\
Cuchilla 02 & 176,85 \\
Cuchilla 03 & 156,57 \\
Cuchilla 04 & 143,60 \\
Cuchilla 05 & 139,21 \\
Cuchilla 06 & 125,96 \\
Cuchilla 11 & 115,04 \\
Cuchilla 12 & 100,75 \\
Cuchilla 13 & 97,54 \\
Cuchilla 14 & 93,89 \\
\hline
\end{tabular}

Elaboración propia

Comparando los valores presentados en la tabla 1 con los tiempos estándar mostrados en la tabla 2, se puede apreciar que la diferencia es significativa, y esto demuestra la existencia de largos tiempos de espera que derivan en largos tiempos de ciclo (tiempo transcurrido entre la primera y la última actividad del proceso por pieza) por lo que se hizo necesario realizar un análisis de las operaciones para determinar cuáles son los factores que afectaban y prolongaban el tiempo de procesamiento.

Identificación de tiempos de ciclo

Con la información proporcionada por la empresa se construyó la tabla 3 con las principales operaciones y el tiempo que tarda la realización de cada una. 
Tabla 3

Duración de las principales operaciones del proceso

\begin{tabular}{|c|c|c|c|c|}
\hline $\begin{array}{l}\text { Estación } \\
\text { de trabajo } \\
\text { (secuencia) }\end{array}$ & Definición de la actividad & $\begin{array}{l}\text { Piezas / hora } \\
\text { (por operador o } \\
\text { equipo) }\end{array}$ & $\begin{array}{c}\text { Número de } \\
\text { operadores o } \\
\text { equipos }\end{array}$ & $\begin{array}{l}\text { Duración (min } \\
\qquad / \text { pza) }\end{array}$ \\
\hline 1 & Operación de acabado & 19,00 & 36 & 0,09 \\
\hline 2 & Inspección dimensional inicial & 28,00 & 12,8 & 0,17 \\
\hline 3 & Tratamiento térmico & 416,67 & 3 & 0,05 \\
\hline 4 & $\begin{array}{l}\text { Preparación para prueba no destruc- } \\
\text { tiva } 1\end{array}$ & 230,00 & 4 & 0,07 \\
\hline 5 & Prueba no destructiva 1 & 190,00 & 5 & 0,06 \\
\hline 6 & Inspección dimensional final & 39,00 & 9.2 & 0.17 \\
\hline 7 & $\begin{array}{l}\text { Preparación para prueba no destruc- } \\
\text { tiva } 2 \text { (etapa 1) }\end{array}$ & 140,00 & 4 & 0,11 \\
\hline 8 & $\begin{array}{l}\text { Preparación para prueba no destruc- } \\
\text { tiva } 2 \text { (etapa 2) }\end{array}$ & 63,00 & 11 & 0,09 \\
\hline 9 & Prueba no destructiva 2 & 188,00 & 4 & 0,08 \\
\hline 10 & Preparación para embarque a cliente & 103,00 & 6 & 0,10 \\
\hline & & & Total & 0,97 \\
\hline
\end{tabular}

Elaboración propia

Determinación de la capacidad máxima

De acuerdo con la tabla anterior y considerando que la capacidad está determinada por la operación con el tiempo más largo por unidad dentro de un proceso (teoría de restricciones), para la producción de productos NG los principales cuellos de botella son dados por las operaciones dimensionales inicial y final. De acuerdo con la tabla 3, cada 0,97 minutos se debía obtener una pieza finalizada. En función de esto, se calcularon los siguientes parámetros referentes al tiempo de ciclo, utilizando la ecuación 1:

$$
\text { Producción diaria máxima }=\frac{\text { Tiempo disponible por día }}{\text { Tiempo de ciclo por unidad }}
$$

$$
\begin{gathered}
\text { Producción diaria máxima }=\frac{(24 \text { horas } / \text { día })(60 \text { minutos } / \text { hora })}{0,97 \text { minutos } / \text { unidad }} \\
\text { Producción diaria máxima }=\frac{1440}{0,97}=1464,54 \text { unidades } / \text { día }
\end{gathered}
$$


Determinación del tiempo de ciclo máximo permisible

Considerando que la proyección de la demanda para el 2020 en ese entonces era de 1030000 piezas al año, que en ese año se tendrían 305 días laborales, con el nivel de producción que se tenía se estimó que no sería posible satisfacer las necesidades del cliente. Con ayuda de la Ecuación 2, se procedió a calcular el tiempo de ciclo máximo permisible (TCMP) que lograría satisfacer las necesidades del cliente para ese año.

$$
\text { TCMP }=\frac{\text { Tiempo disponible por día }}{\text { Número de unidades deseadas por día }}
$$

$$
T C M P=\frac{1440}{(1030000 / 305)}
$$

$$
\text { TCMP }=0,4264 \text { minutos } / \text { unidad }
$$

Lo que indicaba que el tiempo de ciclo debía ser disminuido de 0,97 a 0,4264 ya que es el tiempo de ciclo máximo permisible.

\section{ETAPA 2. Identificación de las principales causas de ineficiencia en la operación}

Ante los resultados del análisis de tiempos, se procedió a realizar monitoreos en el piso de producción haciendo énfasis en las operaciones en las cuales fueron detectados los tiempos más largos (inspección dimensional inicial, tratamiento térmico, inspección dimensional final y prueba no destructiva 2).

Como resultado de entrevistas preliminares con el personal de producción (coordinadores, supervisores y personal operativo) se detectaron algunos factores que podrían estar afectando la capacidad del proceso, los cuales se presentan en el siguiente diagrama de Ishikawa, véase figura 4,

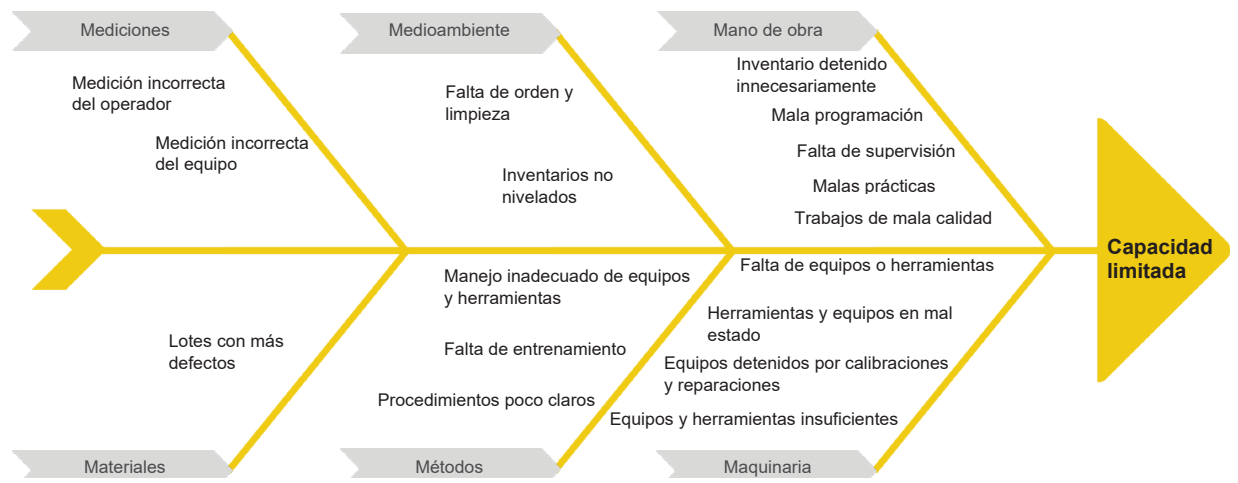

Figura 4. Diagrama de Ishikawa para identificar los factores que podrían estar afectando la capacidad del proceso, de acuerdo con las entrevistas preliminares al personal

Elaboración propia 
Posteriormente y a fin de tener mayor precisión con respecto a la apreciación preliminar, se le solicitó al personal del departamento de producción que, de acuerdo con su experiencia, asignaran una calificación a cada una de las causas identificadas, según su nivel de impacto en la capacidad de producción, con una escala Likert del 1 al 5, en donde 1 significa bajo impacto y 5 significa alto impacto, con la finalidad de cuantificarlas y detectar cuáles son las causas que afectan más significativamente la operación. Los resultados obtenidos se muestran en la tabla 4.

Tabla 4

Causas que afectan la capacidad de producción

\begin{tabular}{|c|c|c|c|c|c|c|c|}
\hline Causa & $\begin{array}{c}\text { Coord. } \\
1\end{array}$ & $\begin{array}{l}\text { Coord. } \\
2\end{array}$ & $\begin{array}{c}\text { Superv. } \\
1\end{array}$ & $\begin{array}{c}\text { Superv. } \\
2\end{array}$ & $\begin{array}{c}\text { Superv. } \\
3\end{array}$ & Total & Porcentaje \\
\hline $\begin{array}{l}\text { Inventario detenido } \\
\text { innecesariamente (malas } \\
\text { prácticas) }\end{array}$ & 5 & 4 & 5 & 4 & 5 & 23 & 11,22 \\
\hline Falta de orden y limpieza & 5 & 4 & 4 & 4 & 3 & 20 & 9,76 \\
\hline $\begin{array}{l}\text { Equipos y herramientas } \\
\text { insuficientes }\end{array}$ & 4 & 4 & 4 & 4 & 4 & 20 & 9,76 \\
\hline $\begin{array}{l}\text { Equipos detenidos por } \\
\text { calibraciones y reparaciones }\end{array}$ & 3 & 4 & 3 & 4 & 4 & 18 & 8,78 \\
\hline $\begin{array}{l}\text { Inventarios no nivelados } \\
\text { (distribución de producto) }\end{array}$ & 4 & 3 & 4 & 2 & 4 & 17 & 8,29 \\
\hline Lotes con más defectos & 3 & 3 & 4 & 2 & 3 & 15 & 7,32 \\
\hline $\begin{array}{l}\text { Equipos detenidos por } \\
\text { distracción del personal }\end{array}$ & 2 & 3 & 3 & 2 & 3 & 13 & 6,34 \\
\hline Trabajos de mala calidad & 2 & 3 & 3 & 2 & 2 & 12 & 5,85 \\
\hline Mala programación & 3 & 1 & 2 & 3 & 2 & 11 & 5,37 \\
\hline Falta de supervisión & 2 & 2 & 1 & 3 & 1 & 9 & 4,39 \\
\hline $\begin{array}{l}\text { Manejo inadecuado de equipos } \\
\text { y herramientas }\end{array}$ & 2 & 2 & 1 & 1 & 2 & 8 & 3,90 \\
\hline Fallo de equipos o herramientas & 1 & 2 & 2 & 1 & 2 & 8 & 3,90 \\
\hline Procedimientos poco diarios & 1 & 1 & 3 & 1 & 1 & 7 & 3,41 \\
\hline Falta de entrenamiento & 1 & 1 & 2 & 2 & 1 & 7 & 3,41 \\
\hline Medición incorrecta del equipo & 1 & 1 & 1 & 2 & 1 & 6 & 2,93 \\
\hline $\begin{array}{l}\text { Herramientas y equipos en mal } \\
\text { estado }\end{array}$ & 1 & 1 & 1 & 2 & 1 & 6 & 2,93 \\
\hline $\begin{array}{l}\text { Medición incorrecta del } \\
\text { operador }\end{array}$ & 1 & 1 & 1 & 1 & 1 & 5 & 2,44 \\
\hline & & & & & Total & 205 & 100,00 \\
\hline
\end{tabular}

Elaboración propia 
Según la tabla 4, se determinó que las principales causas eran:

1. Inventario detenido innecesariamente: Con el personal de acabado, inspección dimensiona inicial y dimensional final se detectó que el personal detenía el producto ya trabajado con el fin de "disminuir el número de vueltas". Estos tiempos de espera son los que aportaban mayor cantidad de tiempo de procesamiento y no agregaban ningún valor al producto.

2. Falta de orden y limpieza: Durante los procesos se utilizan bandejas que, dependiendo del tamaño del producto que estén transportando, pueden almacenar entre dos y ochenta piezas. Era común encontrarse con charolas que tenían piezas de otra operación, esto tenía un efecto negativo ya que el procesamiento del producto se realiza por número de serie, por lo que tener piezas que no corresponden a una operación implicaba destinar tiempo a identificar, separar y moverlas a la operación correspondiente.

3. Equipos y herramientas insuficientes: Faltaban equipos para el procesamiento, especialmente los equipos de medición que se utilizan en las operaciones dimensionales inicial y final para medir las dimensiones de las piezas. La problemática era compleja debido a que, aunque se tenía personal suficiente para mantener un buen flujo de producto, las complicaciones se presentaban dependiendo del inventario de partes disponible que es proporcionado por las plantas suministradoras. La familia de productos NG tiene 10 números de partes diferentes y cada uno de ellos utiliza equipos con características diferentes lo que no permite mucha flexibilidad en el proceso y propicia que en muchas ocasiones grandes inventarios queden en cola en algunos equipos mientras que otros no contaban con material para trabajar.

4. Equipos detenidos por calibraciones y reparaciones: se tenía un problema por la falta de comunicación, coordinación y seguimiento entre los departamentos de calibraciones y producción al momento de retirar los equipos de medición de piso para someterlos a calibraciones programadas. En general, el área de producción no daba un correcto seguimiento y no se tomaban medidas preventivas para el momento en que no se contara con los equipos en piso de producción. Entre los problemas que más comúnmente se presentaban estaban los siguientes:

- El departamento de calibraciones tomaba un equipo para darle mantenimiento sin que el área de producción lo supiera.

- Una vez que finalizaba la calibración, el equipo era regresado a piso de producción sin dar aviso.

- Cuando un equipo entraba a calibración se le detectaban otros problemas, lo que provocaba que se quedara inactivo por más tiempo del previsto. 


\section{ETAPA 3. Propuestas}

En general, las propuestas se engloban en dos grupos:

- Mejora continua y buenas prácticas (propuestas 1, 2, y 4).

- Adquisición de equipos (propuesta 3).

Propuesta 1

Se propuso comenzar a monitorear la frecuencia de liberación de producto como parte de las actividades de supervisión, de tal manera que se pueda tener un flujo constante en la producción hora con hora y de esta forma evitar picos a la mitad del turno y al final de este; así también se planteó la contratación de personal dedicado exclusivamente al movimiento del producto de una operación a la siguiente.

\section{Propuesta 2}

Establecer un mecanismo de nomenclatura de los racks que permitiera identificar fácilmente la operación a la que corresponden, el cliente, la familia de producto y, en casos particulares como los productos NG que cuentan con un mayor volumen de piezas, el número de parte. Se propuso adaptar a cada rack un letrero con información del proceso, como se observa en la figura 5 .

Con este cambio se estima que los cuatro minutos dedicados cada dos horas al movimiento y acomodo de producto en las áreas ya comentadas se pueden reducir a un minuto cada dos horas, lo que para un turno de 8 horas ( 450 minutos sin considerar media hora de comida) representa una disponibilidad de tiempo productivo del 2,4 \%.

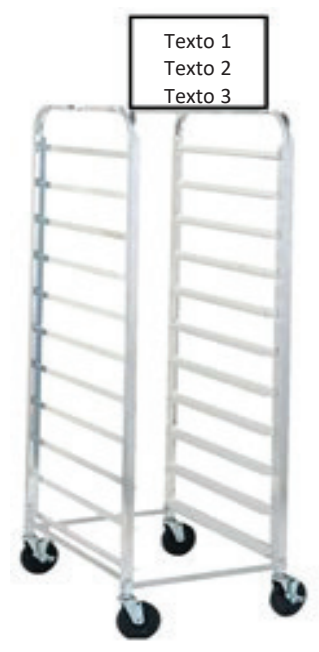

Figura 5. Propuesta de identificación de racks

Elaboración propia 


\section{Propuesta 3}

1. Adquisición de equipos:

La falta de equipos es un factor crucial que impide eliminar la restricción para tener un mejor flujo de producto. Dado que se conocía el volumen de ventas requerido y los requerimientos por número de parte individual, se pudo estimar el equipo requerido para posteriormente determinar el equipo faltante, lo que se presenta en la tabla 5.

Tabla 5

Resultados del cálculo de equipo faltante

\begin{tabular}{cccc}
\hline & \multicolumn{2}{c}{ Equipos de medición } & \\
\hline Modelos & Requeridos & Disponibles & Faltantes \\
\hline Cuchilla 01 & 3 & 2 & 1 \\
Cuchilla 02 & 3 & 2 & 1 \\
Cuchilla 03 & 3 & 2 & 1 \\
Cuchilla 04 & 2 & 2 & 0 \\
Cuchilla 05 & 2 & 2 & 0 \\
Cuchilla 06 & 2 & 2 & 0 \\
Cuchilla 11 & 5 & 2 & 3 \\
Cuchilla 12 & 5 & 3 & 2 \\
Cuchilla 13 & 5 & 3 & 2 \\
Cuchilla 14 & 4 & 2 & 2 \\
\hline
\end{tabular}

\section{Elaboración propia}

Se observa que, para satisfacer la demanda proyectada en las operaciones dimensionales inicial y final, es necesario incrementar la cantidad de equipos de medición en doce unidades.

2. Incremento de horas laborales y adquisición mínima de equipos:

Se planteó que, dado que las operaciones identificadas trabajan seis días a la semana, existía la posibilidad de incrementar su capacidad al trabajar un día más. Con veinticuatro horas más de operación se tendría un incremento de 1/6 sobre la capacidad, 16,67 \% de aumento para estas operaciones. Con un día más de trabajo la cantidad de equipos a adquirir disminuiría, sin embargo, se necesitaría la contratación de personal para cubrir descansos, al igual que el pago de primas dominicales. 


\section{Propuesta 4}

De manera general, se propone la implementación de la cultura del Total Productive Maintenance tan pronto como sea posible, considerando lo que plantea Kiran (2016):

- Maximizar la efectividad de los equipos.

- Establecer un sistema de mantenimiento preventivo minucioso que abarque toda la vida útil del equipo.

- Involucrar no únicamente al departamento de mantenimiento sino al personal operativo, de ingeniería, etcétera.

- Promover el mantenimiento preventivo de los equipos a través de la promoción de este.

De manera específica, se propone implementar un plan de calibraciones (a manera de plan de mantenimiento) en el que se establezcan los tiempos promedio de calibración por equipo, así como las fechas en que los equipos serán retirados de uso para tener fechas tentativas en que serán regresados al piso de producción; de igual forma, se propone que mes a mes se informe sobre las calibraciones correspondientes para que el área de producción esté informada y pueda tomar medidas pertinentes.

A continuación, se presentan de manera resumida las propuestas:

Tabla 6

Principales causas de ineficiencia en la producción identificadas y propuestas de solución

\begin{tabular}{|c|c|c|c|}
\hline \multicolumn{4}{|c|}{ Resumen de propuestas } \\
\hline Causa 1 & $\begin{array}{c}\text { Propuesta } \\
\text { de solución } 1\end{array}$ & Causa 2 & $\begin{array}{c}\text { Propuesta } \\
\text { de solución } 2\end{array}$ \\
\hline $\begin{array}{l}\text { Inventario detenido } \\
\text { innecesariamente } \\
\text { por malas prácticas, } \\
\text { retención de producto } \\
\text { y afectación del flujo } \\
\text { de este. }\end{array}$ & $\begin{array}{c}\text { Monitoreo en la } \\
\text { liberación del producto } \\
\text { y movimiento a las } \\
\text { siguientes operaciones. } \\
\text { Contratación de personal } \\
\text { para movimiento de } \\
\text { producto. }\end{array}$ & $\begin{array}{c}\text { Falta de orden y } \\
\text { limpieza en el acomodo } \\
\text { de producto que rezaga } \\
\text { el producto y atrasa su } \\
\text { procesamiento. }\end{array}$ & $\begin{array}{l}\text { Establecer identificación } \\
\text { de los racks de transporte } \\
\text { y almacenamiento de } \\
\text { producto para no mezclar } \\
\text { diferentes números de } \\
\text { parte correspondientes a } \\
\text { procesos diferentes. }\end{array}$ \\
\hline Causa 3 & $\begin{array}{c}\text { Propuesta } \\
\text { de solución } 3\end{array}$ & Causa 4 & $\begin{array}{c}\text { Propuesta } \\
\text { de solución } 4\end{array}$ \\
\hline $\begin{array}{l}\text { Equipos y } \\
\text { herramientas } \\
\text { insuficientes para el } \\
\text { procesamiento del } \\
\text { producto. Producto } \\
\text { permanece en cola } \\
\text { por largos tiempos. }\end{array}$ & $\begin{array}{c}\text { Adquisición de equipos e } \\
\text { incremento de horas de } \\
\text { operación. }\end{array}$ & $\begin{array}{l}\text { Equipos detenidos } \\
\text { por calibración y } \\
\text { reparaciones. No se } \\
\text { cuenta con todos los } \\
\text { equipos disponibles en } \\
\text { el piso de producción. }\end{array}$ & $\begin{array}{l}\text { Implementación de la } \\
\text { cultura del Total Productive } \\
\text { Maintenance, así como } \\
\text { mejorar la comunicación } \\
\text { interdepartamental } \\
\text { mediante el uso de } \\
\text { controles y notificaciones. }\end{array}$ \\
\hline
\end{tabular}

Elaboración propia 


\section{CONCLUSIONES}

Primero hay que hacer notar que, aunque en un principio no se mostraba mayor interés en la situación del proceso, dada la conformidad que se observó al inicio, a medida que se avanzó en el análisis, particularmente al comparar las proyecciones de demanda con las posibilidades de producción, la perspectiva cambió, pues se hizo patente el riesgo futuro que tenía la maquiladora ante una realidad no vislumbrada.

Durante el desarrollo del presente proyecto se identificaron cuáles son las principales causas que afectan al proceso y se realizaron propuestas para que no solo se mitigaran sus efectos, sino que se agilizara el flujo del proceso, lo que impactará en la flexibilización de éste, dado que podrá producirse una mayor diversidad de productos por día de acuerdo con los requerimientos de los clientes.

En contraste con los tiempos estándar de proceso de una pieza que la empresa había establecido, los cuales son menores a una hora, se encontró que el tiempo de procesamiento promedio de una pieza era mucho mayor al definido, pudiendo tomar más de 170 horas el llevar una unidad desde la actividad inicial hasta el momento en que se empacaba y se preparaba para el cliente. Se identificó que el tiempo efectivo invertido en una pieza debía ser de 0,97 minutos, por lo que, al compararlo con el tiempo real de procesamiento, se encontró que existía una gran afectación al proceso debido a largos tiempos improductivos y de espera que derivaban en largos tiempos de ciclo.

Se identificaron las principales causas de ineficiencia en la operación, entre las que se encontraron las malas prácticas del personal que afectaban el flujo de producto, al no moverlo a las siguientes operaciones, la falta de orden y limpieza en los espacios de trabajo y almacenamiento de producto en proceso, la cantidad de equipos y herramientas que resultaron insuficientes en operaciones críticas, y la falta de comunicación entre los departamentos de calibraciones y producción.

Cabe comentar que, dado que las secuencias de procesamiento no eran iguales para todos los productos, las áreas de trabajo no estaban distribuidas de una manera secuencial, por lo que para futuros trabajos se les sugirió evaluar la reconfiguración del layout de las áreas y actividades del proceso, evaluando la factibilidad de establecer una única secuencia de procesos que permitiera llegar a un nivel de automatización del transporte del producto dentro de la planta.

También cabe recalcar que, a pesar de que se identificaron otras causas además de las presentadas, el criterio de acotamiento a las de mayor impacto en la productividad se realizó mediante el uso de una escala Likert, cuyos resultados se obtuvieron mediante encuestas a los responsables de las diferentes áreas de producción. Para trabajos futuros, se recomendó complementar el proceso de cuantificación del impacto de cada causa mediante un análisis de riesgo, de tal manera que no se trabaje únicamente sobre 
las causas que tengan los mayores efectos sobre la producción sino que también se evalúen desde un enfoque de probabilidad de ocurrencia.

Por último, no está de más comentar que, aunque no fue posible publicar parte de los datos, información y cálculos realizados por razones de confidencialidad, se realizó un ensayo piloto con la mayor parte de las recomendaciones expresadas en el presente documento, lo que evidenció una agilización en el flujo del proceso y una notable mejora en la capacidad de producción, ya que se logró disminuir el tiempo de ciclo del proceso actual en más de $12 \%$ alcanzando un valor de 0,85 minutos por unidad, lo que se podría traducir en un aumento de la producción (al pasar de una producción diaria máxima de 1464,54 unidades/día a 1694,12 unidades/día) lo que conlleva un incremento en la eficiencia del proceso. Se sugirió completar los resultados con un análisis de costos.

\section{REFERENCIAS}

AIRBUS. (9 de junio del 2018). Global Market Forecast 2017-2036. http://www.airbus.com/ aircraft/market/global-market-forecast.html

Arango-Serna, M. D., Campuzano-Zapata, L. F., y Zapata-Cortes, J. A. (2015). Mejoramiento de procesos de manufactura utilizando Kanban. Revistas Ingeniería Universidad de Medellín, 221-234.

Corbetta. (2007). Metodología y Técnicas de Investigación Social. McGraw Hill.

De León, N. (2021). Industria aeroespacial genera 15 mil empleos directos en Mexicali. El Imparcial. https://www.elimparcial.com/tijuana/dinero/Industria-aeroespacialgenera-15-mil-empleos-directos-en-Mexicali-20200525-0003.html

Engineers Edge. (2018). Heat Treatment - Stress Relieving. https://www.engineersedge. com/material_science/heat_treatment__stress_relieving_12886.htm

Hernández-Pólito , A. (2013). Motivación, satisfacción y desempeño laboral en organizaciones públicas y privadas. ANFECA.

Kiran, D. R. (2016). Total Productive Maintenance. En D. R. Kiran, Total Quality Management: Key Concepts and Case Studies (pp. 177-192). Butterworth-Heinemann.

Luna-Puente, R., Guerrero-Aguirre, J. D., Contreras-Amezquita, E., y Moreno-Villanueva, E. (2010). Análisis del proceso de fabricación de cuellos de camisa. TECNOCIENCIA Chihuahua, 39-47.

Molano, A. F., y Materón, C. (2018). Reducción del tiempo de ciclo para el aumento de la productividad en el proceso de elaboración de concentrado para gallinas ponedoras. [Trabajo de grado Ingeniería Industrial, Universidad de San Buenaventura]. 
http://bibliotecadigital.usb.edu.co/bitstream/10819/5545/1/Reduccion_ Tiempo_Ciclo_Molano_2017.pdf

Okutmuş, E., Kahveci, A., y Kartašova, J. (2015). Using Theory of Constraints for Reaching Optimal Product Mix: An Application in the Furniture Sector. Intellectual Economics, 138-149.

Ortiz-Triana, V., y Caicedo-Rolón, A. J. (2015). Procedimiento para la programación y control de la producción de una pequeña empresa. Ingeniería Industrial, 14 (1), 89-104.

Robles Rodríguez, V. M. (2012). Propuesta de mejoramiento del proceso productivo de los cereales en la empresa Big Bran S. A .S. a partir de la implementación de la Teoría de Lean Manufacturing.[Tesis de Grado. Pontificia Universidad Javeriana]. http:// hdl.handle.net/10554/15046

Rocha, H., Pinto, L., y Silva, F. (11 al 14 de junio del 2018). Analysis and Improvement of Processes in the Jewelry Industry. 28th International Conference of Flexible Automation and Intelligent Manufacturing (FAIM2018), Columbus, OH, USA. Procedia Manufacturing, 640-646.

Semprún, A. (2021). La industria aeronáutica superará el COVID en el 2026 tras reducirse un $20 \%$ la flota.ElEconomista.https://www.eleconomista.es/empresas-finanzas/ noticias/11094886/03/21/La-industria-aeronautica-superara-el-Covid-en2026-tras-reducirse-un-20-la-flota.html

Tao, F., Qi, Q., Liu, A., y Kusiak, A. (2018). Data-Driven Smart Manufacturing. Journal of Manufacturing Systems, 48, 157-169. 